\title{
Taking the Lead: A Case Report of a Leiomyoma Causing Duodeno-Duodenal Intussusception and Review of Literature
}

\author{
Louis F. Chai,' Philip M. Batista, and Harish Lavu',*
}

\begin{abstract}
Background: Duodenal masses are rare entities and symptomatic presentation generally is due to abdominal pain or the presence of gastrointestinal bleeding. A number of published case reports in the literature have detailed various neoplasms that have caused an intussusception isolated to the duodenum. This is a particularly unusual phenomenon due to the location and fixation of this portion of the proximal small bowel to the retroperitoneum. We present here a case of duodeno-duodenal intussusception secondary to a leiomyoma.

Case: A 65-year-old Caucasian male presented with intermittent bloody stools and syncope over a 9-month period secondary to a duodenal leiomyoma causing intussusception, which was treated through a pancreaticoduodenectomy.

Conclusion: Intussusception of the duodenum is an uncommon entity and the diagnosis of a leiomyoma should be considered in the setting of a potential mass in the small intestine.
\end{abstract}

Key words: leiomyoma; duodeno-duodenal intussusception; gastrointestinal bleed

\section{Introduction}

Etiologies for gastrointestinal (GI) bleeding commonly include bleeding ulcers, arterial venous malformations, diverticular disease, hemorrhoids, and anal fissures, whereas uncommon causes include Meckel's diverticulum and intussusception. Typically, conventional diagnostic modalities such as physical examination, endoscopy, and radiological imaging yield a cause, although occasionally surgical intervention is required to obtain a diagnosis. Here, we report the first case of a patient with GI bleeding secondary to duodeno-duodenal intussusception caused by a leiomyoma.

\section{Case}

A 60-year-old male presented to the Thomas Jefferson University Hospital in August 2015 with a syncopal epi- sode, fatigue, abdominal pain, and dyspnea on exertion. A review of the patient's records revealed two previous hospitalizations to an outside institution over the prior 9 months for melena and near syncope. A computed tomography (CT) with enterography performed at the outside hospital in November 2014 revealed a $4 \mathrm{~cm}$ intraluminal mass-like density. An esophagogastroduodenoscopy (EGD) with endoscopic ultrasound was performed at that time, which revealed an extrinsic thickened fold of the second portion of the duodenum (D2), concerning for a duodenal duplication cyst. Fine-needle aspiration of the suspected mass revealed normal villous morphology. At the patient's second presentation to the outside hospital in July 2015, a second EGD evaluation revealed a bleeding ulcer in the stomach and a repeat CT showed that the duodenal mass had increased to $5 \mathrm{~cm}$.

\footnotetext{
${ }^{1}$ Rutgers-Robert Wood Johnson Medical School, Piscataway, New Jersey.

${ }^{2}$ Department of Surgery, Jefferson Pancreas, Biliary, and Related Cancer Center, Thomas Jefferson University Hospital, Philadelphia, Pennsylvania.

*Address correspondence to: Harish Lavu, MD, Department of Surgery, Jefferson Pancreas, Biliary, and Related Cancer Center, Thomas Jefferson University Hospital, 1015 Walnut Street, Philadelphia, PA 19107, E-mail: harish.lavu@jefferson.edu

(c) Louis F. Chai et al. 2016; Published by Mary Ann Liebert, Inc. This Open Access article is distributed under the terms of the Creative Commons License (http://creativecommons.org/licenses/by/4.0), which permits unrestricted use, distribution, and reproduction in any medium, provided the original work is properly credited.
} 
At the time of transfer to our institution, the patient reported a recent episode of melena without gross blood. He was found to have a hemoglobin of $6.8 \mathrm{~g} / \mathrm{dL}$ and received two units of packed red blood cells. A CT scan to evaluate the abdominal mass revealed focal dilation and thickening of the third portion of the duodenum (D3) with intussusception of D2 into D3 (Fig. 1). There was also mild intrahepatic biliary dilatation and common bile duct dilatation to $1.2 \mathrm{~cm}$, likely resulting from intussusception of the ampulla of Vater and the distal common bile duct. EGD was performed again, revealing a submucosal lesion along the lateral aspect of D2, causing $80 \%$ narrowing of the lumen and erythematous mucosa overlying the lesion. Colonoscopy showed nonbleeding diverticula and a nonbleeding, benign sessile polyp. Capsule endoscopy was nonrevealing.

Due to the concern for a malignant mass involving the ampullary complex, a pylorus-preserving pancrea-
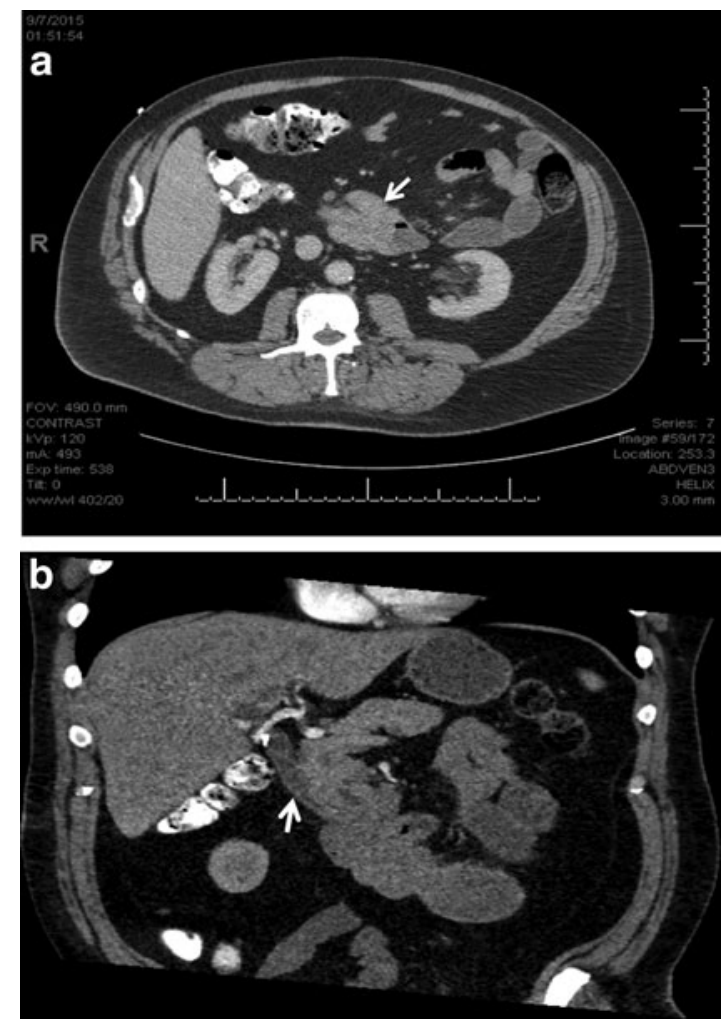

FIG. 1. (a, b) Axial and coronal CT scan slices showing invagination of proximal portion of duodenum into distal (white arrows). CT, computed tomography.

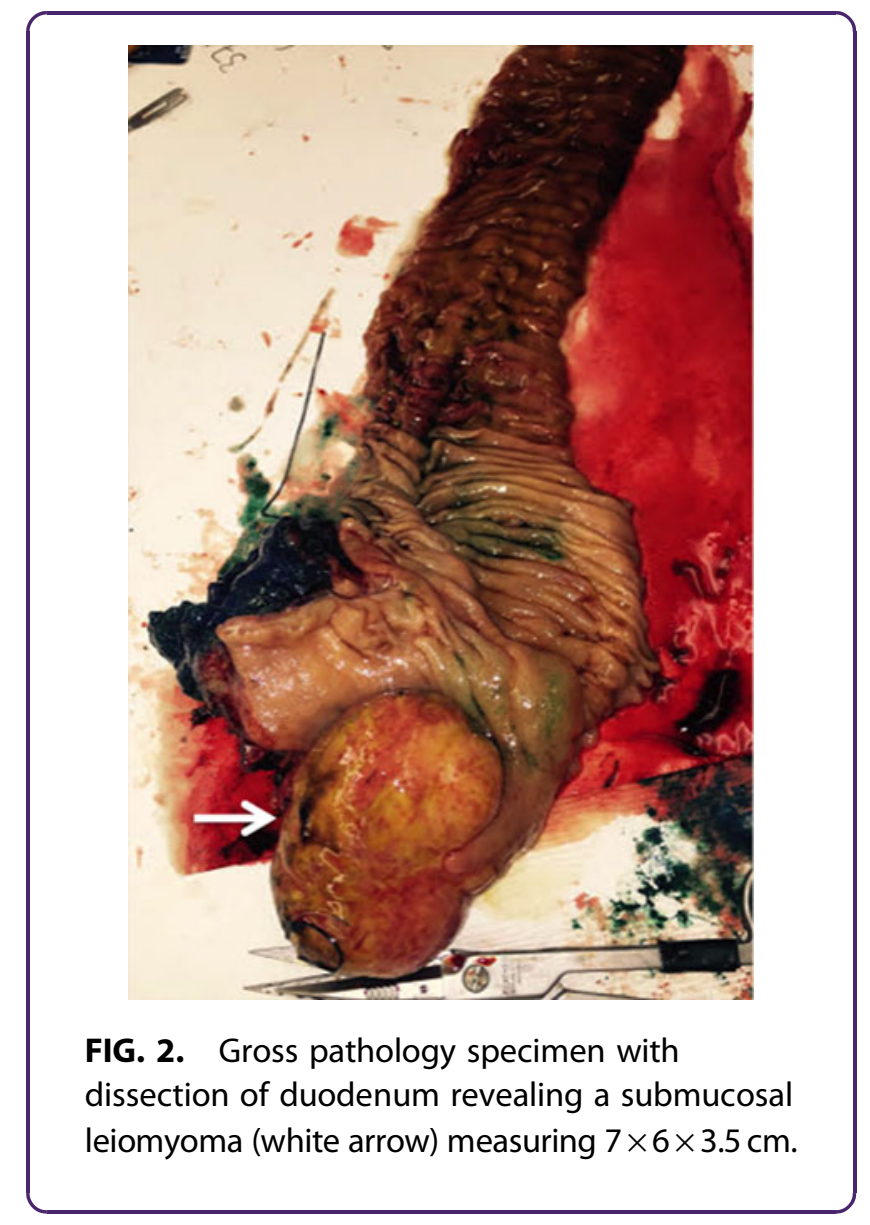

ticoduodenectomy was performed. The specimen was removed and gross pathology revealed a $7 \times 6 \times 3.5-\mathrm{cm}$ duodenal submucosal leiomyoma (Fig. 2). The mass stained positive for actin and desmin and negative for DOG-1, S100, SD34, and ALK-1 (Fig. 3). Negative margins were obtained and none of the 10 harvested lymph nodes were involved with disease. The patient recovered well from surgery, with no further episodes of melena or syncope.

\section{Discussion}

Intussusception is a condition that is more common in children and rarely seen in the adult population. The condition results from the full-thickness invagination of a proximal portion of the bowel into the distal portion. ${ }^{1}$ Presenting symptoms are classically described with the triad of abdominal pain, a palpable abdominal mass, and bloody stools, but all three are clinically found in less than $10 \%$ of the population with intussusception. ${ }^{2}$ More commonly, patients present with nonspecific symptoms including intermittent abdominal pain, nausea, vomiting, and anemia. ${ }^{3}$ Given the wide 


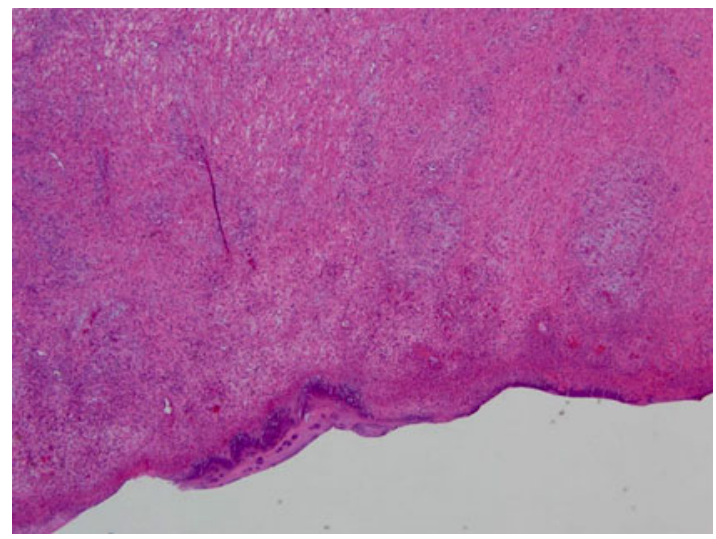

FIG. 3. H\&E stain of pathological specimen. Analysis revealed mitotic figures less than 5 per 50 high-powered field. Specimen stained positive for actin and desmin, negative for DOG-1, S100, CD34, and ALK-1. H\&E, hematoxylin and eosin.

range of diseases with this constellation of symptoms, physicians must have a strong clinical suspicion to make the diagnosis. Work-up may include hemoglobin levels, fecal occult blood tests, cross-sectional imaging (CT or MRI), and endoscopy (EGD, colonoscopy, capsule endoscopy, or push endoscopy). Definitive diagnosis may require operative intervention with surgical exploration of the abdomen.

The most common location for intussusception is the ileocolic junction, followed by ileoileal and colocolic. ${ }^{4}$
Intussusception of the proximal small bowel, especially with the intussusceptum and intussuscipiens isolated to the duodenum, is a rare condition with only 16 reported cases in the literature occurring in 19 total patients (Table 1). ${ }^{1,2,4-17}$ Typically, the anatomic position of the duodenum in the retroperitoneal space and its fixed attachments in this region serve to prevent it from being susceptible to intussusception. However, this condition can occur due to a lead point such as a mass ( $90 \%$ of adults cases) ${ }^{18}$ or secondary to congenital malrotation of the bowel, where the duodenum is more mobile, allowing the intussuception to occur. ${ }^{4}$ The most common etiologies for such lead points include both benign and malignant tumors, lipomas, Brunner's gland hamartomatous polyps, or adenomas. ${ }^{5}$ Although leiomyomas have been known to cause intussusception in other parts of the intestine, our patient represents the first reported case to our knowledge of duodeno-duodenal intussusception secondary to leiomyoma.

Neoplasms of the small bowel as a whole are exceedingly rare, comprising only $1-5 \%$ of all GI neoplasms, with only $1-2 \%$ of these being malignant. ${ }^{19-21}$ The more frequently encountered malignant small bowel tumors are adenocarcinomas, lymphomas, and neuroendocrine tumors. Rarely seen are GI stromal tumors, leiomyosarcomas, and leiomyomas. Although leiomyomas are the most common benign lesions of the small intestine, they are a small proportion of total small intestine neoplasms. At one institution over a 115-year span, fewer than nine leiomyomas were diagnosed

Table 1. English Literature Review Highlighted 16 Reports of 19 Total Patients with Confirmed Duodeno-Duodenal Intussusception

\begin{tabular}{|c|c|c|c|}
\hline Study & Patient age (years) & Presenting symptom(s) & Lead point causing intussusception \\
\hline Vinnicombe and Grundy ${ }^{7}$ & 52 & Abdominal pain, jaundice & Villous adenoma \\
\hline O'Connor et al. $^{8}$ & 32 & Abdominal pain, anorexia, vomiting & Duplication cyst \\
\hline Mishra et al. ${ }^{4}$ & 18 & Abdominal pain and distention, fever, vomiting & None \\
\hline \multirow[t]{2}{*}{ Gupta et al. ${ }^{2}$} & - & - & Adenoma \\
\hline & - & - & Adenoma \\
\hline Limi et al. ${ }^{9}$ & 40 & Abdominal discomfort, melena, diarrhea & Brunner's gland adenoma \\
\hline Abeysekera et al. ${ }^{10}$ & 67 & Abdominal pain, melena & Brunner's gland adenoma \\
\hline Singla et al. ${ }^{11}$ & 43 & Abdominal discomfort, fatigue, generalized weakness & Brunner's gland adenoma \\
\hline Blanchet et al. ${ }^{12}$ & 69 & Abdominal pain, nausea, vomiting & Lipoma \\
\hline Ko et al. $^{13}$ & 43 & Abdominal pain, melena & Duplication cyst \\
\hline Watanabe et al. ${ }^{6}$ & 31 & Abdominal pain & Tubulovillous adenoma \\
\hline Naik et al. ${ }^{1}$ & 35 & Abdominal pain, vomiting & Tubulovillous adenoma \\
\hline Larsen et al. ${ }^{14}$ & 19 & Abdominal pain, nausea, vomiting & Duodenal membrane \\
\hline Sinhal et al. ${ }^{15}$ & 50 & Abdominal pain and distention, vomiting & Tubulovillous adenoma \\
\hline Shakhnovich et al. ${ }^{16}$ & 14 & Vomiting & Duplication cyst \\
\hline Gardner-Thorpe et al. ${ }^{5}$ & 66 & Abdominal pain, lethargy, pruritus & Villous adenoma \\
\hline \multirow[t]{3}{*}{ Pradhan et al. $^{17}$} & - & - & Adenocarcinoma \\
\hline & - & - & Villous adenoma \\
\hline & - & - & Tubulovillous adenoma \\
\hline
\end{tabular}

-, Indicates nonreported data. 
per year, whereas a second report examining 1091 smooth muscle tumors of the small intestine found that only $1 \%$ were truly leiomyomas. ${ }^{21,22}$

Leiomyomas are found most often in the jejunum followed by the ileum and lastly the duodenum. They are characteristically well-defined solitary masses and may appear gray or white. Within the small intestine, they can be found intraluminal, extraluminal, or intramural. $^{22}$ Diagnosis may be difficult despite modern imaging modalities and endoscopy, as was demonstrated in this patient. He suffered from symptoms for nearly 10 months before the diagnosis was confirmed through surgical exploration. In the setting of intermittent GI bleed and abdominal pain with no easily identifiable source, it is important to consider the diagnosis of small bowel intussusception in an adult due to a neoplasm.

\section{Conclusion}

This case report is that of GI bleeding due to a rare duodeno-duodenal intussusception secondary to a small bowel leiomyoma. We emphasize the importance of a keen awareness for this diagnosis and thorough work-up. In this scenario, surgical intervention may prove to be both diagnostic and therapeutic as demonstrated here.

\section{Acknowledgment}

No funding was needed for the writing of this case report or review of literature.

\section{Author Disclosure Statement}

No competing financial interests exist.

\section{References}

1. Naik BM, Arjun N, Kudari A. A case of duodeno-duodenal intussusception. J Med Dent Sci. 2015;35:6138-6142.

2. Gupta V, Doley RP, Bharathy KGS, et al. Adult intussusception in Northern India. Int J Surg. 2011;9:297-301

3. Chen XD, Yu YY, Yang L, et al. Duodenal intussusception due to a giant neuroendocrine carcinoma in a patient with Peutz-Jeghers syndrome: case report and systematic review. Eur J Gastroenterol Hepatol. 2012;24:722-726.

4. Mishra SK, Mohapatra KC, Panda G, et al. Pseudocyst of pancreas secondary to duodeno-duodenal intussusception: rare presentation of rare disease. Int J Innov Res Adv Stud. 2013;2:667-672.

5. Gardner-Thorpe J, Hardwick RH, Carroll NR, et al. Adult duodenal intussusception associated with congenital malrotation. World J Gastroenterol. 2007;13:3892-3894.

6. Watanabe F, Noda H, Okamura J, et al. Acute pancreatitis secondary to duodenoduodenal intussusception in duodenal adenoma. Case Rep Gastroenterol. 2012;6:143-149.

7. Vinnicombe S, Grundy A. Case report: obstructive jaundice secondary to an intussusception duodenal villous adenoma. Clin Radiol. 1992;46:63-65.

8. O'Connor PA, McGrath FP, Lane BE. Duodenal intussusception secondary to internal duodenal duplication. Clin Radiol. 1999;54:69-70.

9. Limi L, Liew NC, Badrul RH, et al. Duodenal intussusception of Brunner's gland adenoma mimicking a pancreatic tumor. Med J Malaysia. 2010;65:313-314.
10. Abeysekera WYM, de Silva WDD, Pragatheswaran $P$, et al. Brunneroma presenting with radiologic features of duodeno-duodenal intussusception. Sri Lanka J Surg. 2012;30:36-38.

11. Singla $R$, Bharti $P$, Jain $R$, et al. Giant Brunner gland adenoma manifesting as iron deficiency anaemia and intussusception. Natl Med J India. 2010;23:376-377.

12. Blanchett $M C$, Arnal E, Paparel $P$, et al. Obstructive duodenal lipoma successfully treated by endoscopic polypectomy. Gastrointest Endosc. 2003;58:938-939.

13. Ko SY, Ko SH, Ha S, et al. A case of a duodenal duplication cyst presenting as melena. World J Gastroenterol. 2013;19:6490-6493.

14. Larsen PO, Ellebaek MB, Pless T, et al. Acute pancreatitis secondary to duodeno-duodenal intussusception caused by a duodenal membrane in a patient with intestinal malrotation. Int J Surg Case Rep. 2015;13: 58-60.

15. Sinhal M, Kang M, Narayana S, et al. Duodenoduodenal Intussusception. J Gastrointest Surg. 2009;13:386-388.

16. Shakhnovich V, Colombo J, Desai AA, et al. Rare presentation of pancreatitis secondary to intussusception of duodenal duplication cyst, a pediatric case report. J Ped Surg Case Reports. 2014;2:527-529.

17. Pradhan D, Kaur N, Nagi B. Duodenoduodenal intussusception: report of three challenging cases with literature review. J Can Res Ther. 2015;11:1031.

18. Ko SF, Lee TY, Ng SH, et al. Small bowel intussusception in symptomatic pediatric patients: experiences with 19 surgically proven cases. World J Surg. 2002;26:438-443.

19. Serour F, Dona G, Birkenfeld S, et al. Primary neoplasms of the small bowel. J Surg Oncol. 1992;49:29-34.

20. Chaaya A, Heller SJ. Introduction to small bowel tumors. Tech Gastroint Endosc. 2012;14:88-93.

21. Blanchard DK, Budde JM, Hatch GF, et al. Tumors of the small intestine World J. Surg. 2000;24:421-429.

22. Xynopoulos D, Mihas AA, Paraskevas E, et al. Small bowel tumors. Ann Gastroenterol. 2002;15:18-35.

Cite this article as: Chai LF, Batista PM, Lavu H (2016) Taking the lead: a case report of a leiomyoma causing duodeno-duodenal intussusception and review of literature, Case Reports in Pancreatic Cancer 2:1, 19-22, DOI: 10.1089/crpc.2016.0001.

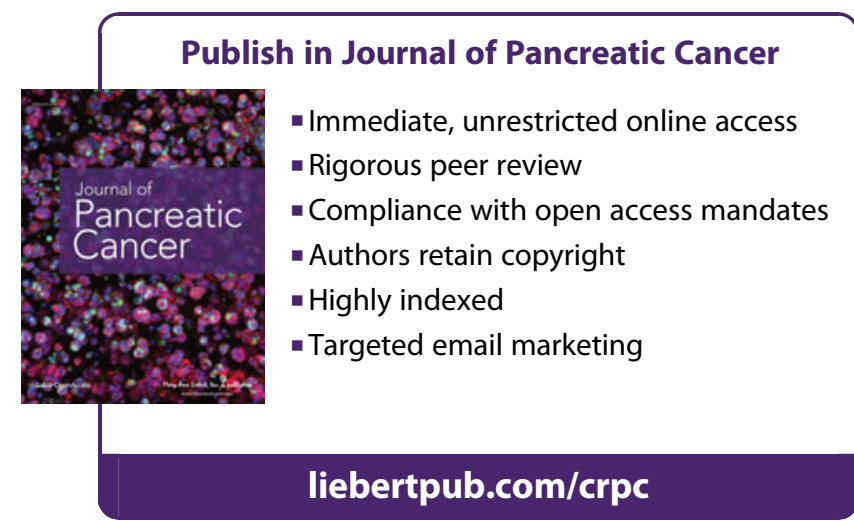

